\title{
Presentación a Estudios de Interfaz Sintaxis-Pragmática
}

\section{Objetivos del volumen}

El estudio la interfaz sintaxis-pragmática, en particular en su vertiente conocida como "estructura informativa" ha experimentado en décadas pasadas una verdadera eclosión. Si bien el interés por las funciones pragmáticas de los componentes oracionales puede rastrearse bastante más atrás (cf. von Heusinger 1999), a partir de años noventa, y de la publicación de estudios como los de Vallduví (1992) y Lambrecht (1994), la literatura sobre estructura informativa se ha multiplicado y ha alcanzado un altísimo grado de riqueza conceptual y sofisticación analítica. Sin embargo, y en particular tratándose de categorías ligadas al lenguaje en uso, es de extrañar la relativa escasez de trabajos basados en datos naturales analizados en su contexto de aparición. Por otra parte, en relación con las categorías empleadas, es notable tanto la gran heterogeneidad de lo que se ha interpretado como "tópico" o "foco" (categorías que tienen un lugar central en los estudios sobre estructura informativa) como la constelación de diversos significados pragmáticos codificados en la morfosintaxis oracional, cuya exclusiva interpretación en términos de marcadores de tópico o foco puede resultar empobrecedora.

En este contexto, las contribuciones que se presentan en este volumen comparten dos principios básicos: (i) la identificación del significado pragmático de un morfema o construcción debe estar sustentada por suficiente evidencia explícita de sus contextos reales de uso; y (ii), los significados pragmáticos relevantes para el estudio de la interfaz sintaxis-pragmática exceden la codificación de estructura informativa en el ámbito oracional (especialmente en términos exclusivos de tópico o foco) para incluir, por ejemplo, las funciones discursivas que distintas construcciones puedan tener en la organización del texto.

Nuestro objetivo es aportar, desde estas premisas, a la consolidación de los estudios de interfaz sintaxis-pragmática disponibles para el público hispanoparlante, a través de contribuciones que sean relevantes para los especialistas pero que también, gracias a la justificación de los postulados teóricos y perspectivas de análisis adoptados en cada caso, motive el interés de estudiantes e investigadores no especializados en el área.

Valeria A. Belloro, Universidad Autónoma de Querétaro, Querétaro, México 
Creemos que los capítulos que integran este volumen presentan un panorama suficientemente abarcador de la riqueza de valores pragmáticos asociados con construcciones particulares: distintos tipos de “tópicos” y “focos”, pero también valores de contra expectativa, así como ligados a la organización de los turnos de habla, la organización del discurso, o a la reconceptualización de los participantes. Al mismo tiempo, el volumen muestra un interesante abanico de estrategias que sirven para codificar significados pragmáticos gramaticalizados: prosodia, marcadores discursivos, variaciones en el orden de constituyentes, cláusulas subordinadas, estructuras hendidas, quiásticas y existenciales, entre otras. Por su parte, las lenguas bajo estudio comprenden variedades peninsulares y americanas del español, pero también lenguas de América como el zapoteco, el toba y el enlhet. Finalmente, los corpus empleados incluyen conversación espontánea, habla dirigida a niños, narrativas orales, discurso político y textos periodísticos y literarios, así como un diseño experimental. En este sentido, confiamos en que el volumen evidencia una parte representativa de la diversidad de enfoques que los abordajes funcionalistas basados en análisis de datos naturales son capaces de aportar al estudio de la interfaz sintaxis-pragmática.

\section{2 ¿De qué hablamos (en este volumen) cuando hablamos de interfaz sintaxis-pragmática?}

Como mencionamos arriba, dentro del dominio de la interfaz sintaxis-pragmática, uno de los aspectos que ha acaparado mayor atención es el estudio de la estructura informativa; es decir, de la articulación de los constituyentes oracionales de acuerdo con funciones pragmáticas como tópico (/comentario) y foco (/trasfondo). Varios de los capítulos de este volumen introducen y aplican estas categorías, y tanto las discusiones y análisis que en ellos aparecen como las referencias que incluyen pueden servir al lector no especializado para familiarizarse con las nociones básicas. Por ello, no es nuestra intención aquí revisar estos conceptos, sino comentar algunos aspectos que tienen particular relevancia para los capítulos que se presentan y pueden servir como telón de fondo de los análisis que siguen.

En este sentido, en relación con la noción de tópico, en primer lugar, es necesario tener en cuenta que en uno de sus usos típicos sirve para denotar a las entidades que aparecen codificadas en un texto, independientemente de su función sintáctica o pragmática. Esta es la noción de tópico detrás de muchos estudios de "continuidad tópica” (Givón 1983) que rastrean, entre otros fenómenos, la relación que existe en las formas de codificación de los participantes en el discurso y su "continuidad" en el texto, generalmente evaluada en 
términos de la distancia en cláusulas que media entre una expresión referencial y su antecedente, o su persistencia en cláusulas subsecuentes. Desde esta perspectiva, "tópico" es en gran medida equivalente a "referente discursivo" (Karttunen 1976), y una oración puede tener tantos tópicos como referentes discursivos aparezcan en ella. Esto puede ilustrarse con el siguiente ejemplo:

\section{(1) a. Habia una vez un gigante amable $_{j}$ \\ b. El gigante , cuidaba a los habitantes de la ciudad $_{k}$ \\ c. y $\emptyset_{j}$ cultivaba un pequeño huerto.}

En (1a) aparece un tópico -discontinuo, en tanto denota un participante discursivo nuevo- codificado con una frase nominal indefinida ("un gigante amable"). Pero en (1b), por su parte, habría dos tópicos: uno continuo, en tanto retoma un referente codificado en la cláusula inmediata precedente, y que es codificado con una frase definida ("el gigante”), y otro discontinuo, en tanto introduce un participante nuevo, codificado con una frase nominal definida ("los habitantes de la ciudad"). Generalmente, los tópicos continuos se codifican con frases definidas simples (como el sujeto de (1b)) o formas atenuadas (como el sujeto de (1c)), mientras que los tópicos discontinuos, dependiendo de factores como su identificabilidad o unicidad, pueden codificarse con frases nominales definidas (como el objeto de (1b)) o indefinidas (como el objeto de (1c)). Sin embargo, el grado de continuidad de los participantes discursivos no solamente motiva el tipo de expresión referencial que se utiliza para codificarlos, sino que también típicamente se asocia con distintas funciones sintácticas, o incluso con ciertas construcciones particulares, y varios capítulos de este volumen estudian estos aspectos.

Más allá de la noción de "tópico" como equivalente a referente discursivo, el término también es usado con un sentido más específico, para designar ya no a los distintos referentes codificados en una oración sino particularmente a aquel que sirve de anclaje a la predicación pragmática. En este sentido, la noción de tópico designa una relación de tematicidad (aboutness). Bajo esta interpretación, (1b) tiene solamente un tópico (el gigante), ya que la oración trata acerca de él, y no de los habitantes de la ciudad.

En realidad, en este sentido de tematicidad, el gigante amable no solamente es el tópico de (1b), sino de hecho de toda la secuencia. Más aún, es plausible que el resto del texto trate de algo que hizo o de algo que le pasó al gigante. Es decir, podemos asignar funciones de tópico temático no solamente a nivel de la oración, sino también de la secuencia o del discurso en su conjunto (van Dijk 1981), y una de las cuestiones a resolver es qué características presenta cada uno. Podemos distinguir, por ejemplo, tópicos temáticos "nuevos" (la primera de las varias menciones del gigante amable en (1a)), o “dados” (la subsecuente mención del gigante 
que aparece en (1b) y (1c)), así como "resuntivos" (que reaparecen en el texto después de estar ausentes) o "subordinados” (que denotan un referente relacionado con un tópico previo).

También es necesario tener en cuenta que la función de tópico puede asignarse ya no al referente discursivo que sirve de anclaje a la predicación pragmática sino particularmente a la expresión lingüística que lo codifica. Es decir, "tópico" es entendido como una función (pragmática) desempeñada por constituyentes oracionales, del mismo modo que las funciones sintácticas de sujeto, objeto, etc. La divergencia entre ambas extensiones del término es evidente en el clásico ejemplo de Reinhart (1981):

\section{(2) A: Who did Felix praise? \\ B: Felix praised HIMSELF.}

En efecto, si la función de tópico se asigna a los referentes, tendríamos que concluir que en la respuesta de $\mathrm{B}$ el referente Felix es a la vez tópico y foco. Por el contrario, si la función de tópico (o foco) se asigna a los constituyentes oracionales, es posible asignarle al constituyente "Felix" la función pragmática de tópico y al constituyente "himself" la función pragmática de foco, del mismo modo que se asigna al primero la función sintáctica de sujeto y al segundo la función sintáctica de objeto.

A partir de esta distinción entre referente topical y expresión topical también es posible reconocer que una oración puede predicarse pragmáticamente de un referente (topical) y al mismo tiempo no tener un tópico sintáctico. Esto puede ilustrarse con el ejemplo de (3)

\section{(3) A: ¿Qué me cuentas de Paula? \\ B: (Paula) Acaba de tener un hijo.}

En (3B) el sujeto sintáctico ("Paula"), de aparecer, cumpliría la función pragmática de tópico. Pero puede también omitirse (y esto es de hecho lo más probable, ya que denota a un referente activo). En este caso, la respuesta ya no tendrá un constituyente sintáctico que cumpla la función de tópico, aunque es evidente que seguirá habiendo un referente topical. Sin embargo, hay estructuras en las que no solamente no aparece un constituyente con función de tópico, sino que tampoco es evidente cuál es el referente que sirve de tópico. Por ejemplo, antes de la respuesta de (4B) no hay ningún referente que pueda construirse a priori como centro de interés y base de la predicación: no sabemos si lo que pasó tiene que ver con el clima, la situación política, el perro o con cualquier otra entidad. 

(4) A: ¿Qué pasó?
B: ¡El perro se comió mi almuerzo!

Algunos autores consideran a estas estructuras como de foco oracional -es decir, sin tópico- (Lambrecht, 1994), pero otros proponen que en estos casos es necesario postular un tópico implícito, “de escenario" (stage topic), que indica los parámetros espacio-temporales a los que aplica la predicación; es decir, el aquí y ahora del enunciado (Erteschik-Shir 1997). Desde esta última perspectiva, los tópicos de escenario serán un tipo de estrategia de marcación de tópico, que alternará con otros: por ejemplo, la omisión de la frase tópico (topic-drop) en la versión con sujeto tácito de (4B), o la dislocación a la izquierda (5B).

\section{(5) A: ¿Supiste que Paula se fue de vacaciones a Brasil y terminó quedándose a vivir? \\ B: Sí, esa historia me la contaron la otra noche.}

En este sentido, no solamente es posible estudiar qué tipo de referentes codifican las frases con función de tópico (por ejemplo, sin son referentes continuos, discontinuos o nuevos), sino también qué características estructurales presentan las expresiones topicales (si están presentes o ausentes, dentro o fuera de la cláusula, qué funciones sintácticas cumplen, con qué tipos de frases se asocian, o qué otros rasgos gramaticales determinan). Y, por supuesto, también es necesario estudiar la relación entre ambos dominios.

Por ejemplo, hay razones para analizar la expresión topical "esa historia” en (5B) como una dislocación a la izquierda; es decir, como un constituyente que ocurre fuera del margen izquierdo de la cláusula. En español, una de las muestras de esta dislocación del objeto (o “topicalización”) es que en la cláusula aparece un clítico correferencial que es el que cumple la función gramatical de objeto, ya que la frase nominal sirve una función exclusivamente pragmática (de tópico). Sin embargo, existen casos en los que estos objetos topicalizados no determinan la aparición de un clítico correferencial. Por ejemplo, el clítico es de hecho agramatical con nombres escuetos singulares (6a), y opcional con plurales (6b).
(6) a. Alcohol no $\left({ }^{*}\right.$ lo) toma.
b. Historias (las) cuenta todo el tiempo.

Sin embargo, se ha argumentado a través de una serie de criterios sintácticos que casos como los de (6) también constituyen instancias de dislocación a la izquierda (Casielles 2004). La pregunta que surge aquí, entonces, es si al asignar una misma caracterización sintáctica debemos también reconocer una misma función 
pragmática. Leonetti (2011, 114), por ejemplo, observa que "la sintaxis de la dislocación impone el requisito de que la expresión indefinida cuente como tópico”. Aceptar esta premisa, sin embargo, impone a su vez la necesidad de revisar la caracterización funcional de "tópico" como (el constituyente que denota a) el referente que sirve de anclaje a la predicación, en la medida en que sería difícil postular que (6a) es un enunciado "respecto de alcohol", o que (6b) "trata de historias". Una solución, entonces, es proponer que no todos los tópicos pueden ser precedidos por expresiones como "respecto de x", y por lo tanto estas expresiones no sirven para identificar la función de tópico (Olarrea 2012, 612). Un enfoque alternativo, por supuesto, sería evitar caracterizar como tópicos casos como los de (6). Y aún otra posibilidad es reconocer distinto tipo de tópicos, algunos (prototípicos) que pasan la prueba de aceptar expresiones del tipo de "respecto de x", y otros que no, y cuya adscripción a la clase es necesario justificar por otros medios.

También sujeta a distintas interpretaciones, pero relativamente menos conflictiva que la noción de tópico, la categoría de "foco" puede definirse, en términos amplios, como aquella función pragmática que se asigna al elemento que hace que la proposición resulte informativa. Esta caracterización, sobre la que existe (con distintas formulaciones) un consenso relativamente generalizado (pero cf. Matić 2015 para una revisión clara y concisa de otras alternativas), por supuesto no dice nada respecto de cuáles son las estrategias con las que se asigna la función de foco, o sobre qué clase de elementos, o de qué tipo de informatividad agrega, y son estos justamente los aspectos que interesa investigar y donde emerge la riqueza de enfoques teóricos y metodológicos posibles.

Pero ¿cómo se definen entonces las funciones de tópico o foco? ¿Están basadas en criterios nocionales y funciones pragmáticas, o se deducen de criterios formales asociados a cierto tipo de codificación morfosintáctica? Y, por otra parte, ¿cuál es su validez tipológica? Ante la diversidad de propuestas coexistentes, creemos que más que abogar por una caracterización deductiva y universal, podemos considerar estas funciones como "categorías radiales" (Lakoff 1987) organizadas alrededor de prototipos centrales, con los cuales los otros miembros de la categoría compartirán más o menos rasgos. Creemos que solo en este sentido una definición apriorística de este tipo de funciones puede seguir siendo útil como andamiaje, quizás provisional, a partir del cual ir construyendo caracterizaciones más ajustadas de los efectos pragmáticos específicos que expresan distintas construcciones en distintas lenguas. Por otra parte, coincidimos con propuestas recientes en el sentido de que el uso hegemónico de las categorías de "tópico" y "foco" puede enmascarar otro tipo de valores pragmáticos, presentes tanto en la articulación de funciones a nivel oracional como asociados a la organización del discurso (Matić y Wedgwood 2013, Ozerov 2015). 
Tomando en cuenta todas estas consideraciones, en este volumen hemos enfatizado el análisis a partir de datos espontáneos, la caracterización de las funciones pragmáticas a partir de la discusión de contextos reales de uso, y un abordaje que incorpora la consideración de funciones que exceden la articulación de la estructura informativa a nivel oracional. Y ahora sí, luego de esta puesta en contexto general, lo que sigue es presentar en más de detalle los aspectos específicos que trata cada capítulo.

\section{Contenido del volumen}

En “Sobre una estrategia topicalizadora del español”, Cristina Sánchez López analiza desde la perspectiva de la interfaz sintaxis-pragmática una particular estructura del español: el grupo nominal del tipo de “(un) + N + oración de relativo”. El interés de estas estructuras es múltiple: en primer lugar, presentan como enunciado autónomo una secuencia que no es oracional sino nominal; en segundo lugar, evidencian la posibilidad de un uso anafórico de los indefinidos, que retoman un referente previamente mencionado; finalmente, muestran que las subordinadas de relativo -típicamente asociadas a valores topicales- cumplen, en estas estructuras, una función focal. A partir del análisis detallado de datos del Corpus del Español Actual (CREA) y del Corpus del Español del Siglo XXI (CORPES), la autora muestra los distintos tipos de relaciones anafóricas que el indefinido establece con su antecedente, desde la repetición del mismo sustantivo hasta anáforas conceptuales ligadas al uso de hiperónimos o encapsuladores, y el rango de distancias sintácticas y discursivas que puede mediar entre ambos. En todos los casos, sin embargo, la función pragmática es la misma: el sustantivo precedido por el indefinido se comporta discursivamente como un tópico (con propiedades similares a los tópicos dislocados), sobre el que la relativa restrictiva aporta información focal. Las características particulares de esta estrategia topicalizadora con una anáfora indefinida la hace particularmente útil para mantener la atención del oyente o lector, quien debe interpretar la nueva información como parte integrante de la unidad discursiva previa. En este sentido, señala la autora, estas construcciones cumplen también una función en la organización textual, en la medida en que contribuyen a crear y mantener unidades de discurso más extensas.

En "Orden de palabras, posición del objeto y estructura de la información en toba/ qom (Guaycurú)”, Paola Cúneo y Cristina Messineo estudian la interfaz sintaxis-pragmática en toba, una lengua guaycurú hablada alrededor de la región del chaco argentino. El toba es una lengua de orden relativamente estable AVO en las cláusulas transitivas y VS en las intransitivas, si bien ciertos factores 
pragmáticos pueden motivar alteraciones en el orden básico. En este capítulo, las autoras se centran en las construcciones transitivas con objeto preverbal. Para ello, estudian un corpus obtenido de primera mano, formado por narrativas, consejos y rogativas, así como por material elicitado a partir de la "Historia de la pera" (Chafe 1980) y a través del Questionnaire for Information Structure (QUIS) (Skopeteas et al. 2006). Esta gran diversidad de fuentes y recursos les permitió comprobar la existencia de tres tipos distintos de construcciones de orden marcado: las construcciones con verbo impersonal $q a-/ q o-$, las construcciones con el adverbio ndoteek, y las escisiones de objeto. Las autoras discuten los aspectos sintácticos y pragmáticos que caracterizan a cada construcción y muestran que las construcciones impersonales y las construcciones con ndoteek constituyen casos de preposicionamiento (Ward y Birner 2004), las primeras para marcar tópicos y las segundas focos restrictivos (Dik et al. 1981), mientras que, en la estructura escindida, biclausal, el objeto escindido marca focos de reemplazo (Dik et al. 1981).

En "Las funciones pragmáticas y la posición del sujeto en español”, Raquel Hidalgo Downing estudia las funciones discursivas de los sujetos preverbales y postverbales en un corpus formado por diez textos, escritos y orales, procedentes de distintos géneros discursivos. Aplicando la taxonomía de Dik $(1989,1997)$, la autora distingue las funciones de tema dado, tema nuevo, tema subordinado, tema resuntivo y oraciones sin tema, discutiendo e ilustrando cada una. Estas funciones se aplican al análisis de los textos, con el fin de evidenciar la relación entre la posición del sujeto y los movimientos discursivos de cambio y continuidad temática. El análisis muestra que los sujetos preverbales exhiben un abanico de funciones temáticas, pero sirven principalmente para articular los temas oracionales en secuencias que se proyectan en el discurso. Los sujetos postverbales, por su parte, se especializan en la introducción de predicados no temáticos, que expresan acontecimientos de forma global y, en segundo lugar, en la introducción de temas nuevos que pasan a ser temas de oraciones subsecuentes. La discusión detallada de las distintas categorías y su aplicación a datos concretos que se analizan tanto cualitativa como cuantitativamente busca también servir como modelo de análisis de la articulación de las funciones pragmáticas oracionales con las ligadas a la progresión del discurso.

En "Más allá de tópico y foco: Construcciones quiásticas y el morfema LA en el zapoteco del Istmo", Juan José Bueno Holle estudia la codificación de la estructura informativa en esta lengua zapotecana de la región de Juchitán (México). El análisis de las construcciones de foco de predicado, oracional y estrecho (Lambrecht 1994), permite caracterizar al zapoteco del Istmo en términos de la tipología de Van Valin (1999) como una lengua en la que tanto la estructura focal como el orden de constituyentes son relativamente flexibles. Con base en esta 
caracterización inicial, el autor se vale de un corpus de narrativas y conversaciones recolectadas en trabajo de campo para analizar dos estructuras particulares con funciones pragmáticas que exceden lo oracional: una "construcción quiástica”, que consiste en la secuencia de un foco de predicado y un foco estrecho, y sirve para marcar el fin del turno del hablante y ceder la palabra al interlocutor y, en segundo lugar, construcciones con la partícula la, que marcan contenidos presupuestos entre los participantes de un acto de habla y hacen explícita la organización de la conversación.

En "Tópicos y focos iniciales en el español hablado: funciones pragmáticas y correlatos formales”, Christoph Hülsmann introduce las nociones de familiaridad asumida de los referentes discursivos, la articulación tópico-comentario, y la articulación foco-transfondo como las tres subdimensiones que componen la estructura de la información. De acuerdo con estas caracterizaciones, e incorporando también consideraciones de nivel prosódico, el autor revisa las funciones pragmáticas de los constituyentes preverbales en un corpus de diálogos espontáneos de hablantes de Madrid y Segovia (C-Oral Rom, Cresti y Moneglia 2005). Respecto de los constituyentes iniciales con función de tópico, se distinguen tres estrategias de topicalización, caracterizadas en virtud del grado de integración sintáctica del elemento topicalizado respecto del resto de la cláusula: los chinese style topics, los hanging topics, y los tópicos dislocados a la izquierda, y se discute la ocurrencia de distintos casos en el corpus. En relación con los constituyentes iniciales con función de foco, se argumenta que pueden tener tanto un valor correctivo como informativo. Al mismo tiempo, el autor cuestiona la habitual dicotomía entre foco informacional y foco contrastivo, analizando ejemplos en los que se combinan los dos tipos, así como casos que clasifica como mirativos y exclamativos, y dan cuenta de la riqueza de matices funcionales que emerge del análisis del discurso espontáneo.

En “Orden de los argumentos en enlhet”, Hannes Kalish presenta un estudio del funcionamiento de la interfaz sintaxis-pragmática esta lengua mascoyana del chaco paraguayo. El autor muestra que, en enlhet, la estructura informativa de la cláusula es codificada prioritariamente a través del orden de constituyentes, y a partir de una distinción básica entre cláusulas con verbo inicial y cláusulas con argumento inicial. Mientras que las primeras presentan típicamente una estructura de tópico-comentario, las segundas se emplean para marcar distintos tipos de focos. La riqueza de matices funcionales expresado por las distintas construcciones de este tipo resulta en una taxonomía que parte las propuestas de Dik (1981) y Sasse (1995) para ampliarlas con categorías adicionales, y en la que los ejemplos ilustran distintos tipos de focos estrechos (completivo, de insistencia, de verificación, de precisión, entre otros) y amplios (anunciativo, explicativo, elaborativo, entre otros). A través del análisis detallado de las situaciones 
comunicativas específicas en las que emergen los distintos usos, el autor muestra que, además de sus diferencias en términos de alcance, foco estrecho y foco amplio responden a condiciones asertivas distintas y se asocian con contextos discursivos particulares. El rango de significados emergente lleva a concluir que las funciones pragmáticas de tópico y foco deben ser complementadas con el análisis de las funciones discursivas que las distintas estructuras analizadas sirven para codificar.

En "Conformación de los marcadores discursivos en la historia del español. El caso de al contrario y a propósito”, Rodrigo Flores Dávila estudia dos marcadores discursivos del español desde el punto de vista de su evolución diacrónica. A partir del análisis de una base de datos que abarca ejemplos desde el siglo XIII al XXI, el autor muestra cómo las construcciones con a más contrario y propósito experimentaron un proceso de fijación léxica que desembocó en la ampliación de contextos sintácticos de aparición para, finalmente, desencadenar la ampliación de contextos pragmáticos de uso. Más allá de las particularidades que se describen para cada marcador, el artículo da cuenta de un proceso de evolución diacrónica similar, pautado por tres grandes etapas funcionales: modificador sintáctico, modificador pragmático y relacionante pragmático. En esta última etapa es que estas formas adquieren su función plena como marcadores discursivos. Al contrario, con una función de contra-argumentación, es empleado para refutar información previa; a propósito, como digresor, introduce información que se añade con valor marginal. La descripción de estas formas desde una perspectiva histórica revela las condiciones que motivaron el cambio gramatical que resultó en los valores pragmáticos actuales.

En "El focalizador aspectual guaraní hína en español paraguayo (jopara): significado, sintaxis y pragmática”, Élodie Blestel aborda la trayectoria de un marcador de aspecto progresivo del guaraní (una lengua tupí-guaraní del Paraguay) y analiza, a partir de un corpus de datos de prensa escrita, los valores con los cuales ha sido incorporado al español paraguayo jopara actual. La autora argumenta que, en esta variedad de habla, hína ya no codifica el aspecto progresivo del predicado (ni, como también ha sido propuesto para los predicados estativos, es un marcador de relevancia). Se propone, sin embargo, que su valor original de marcador de aspecto progresivo se recupera en el dominio más abstracto de la construcción dialógica del significado. Así, hína es caracterizado como un marcador de focalización atencional que, al "aspectualizar” la atención del interlocutor, lo invitar a revisar sus creencias, dando origen a valores pragmáticos de contraexpectativa. De acuerdo con el análisis, esto es particularmente evidente a la luz de ejemplos en los que hína aparece junto a predicados estativos, casos en los que el tiempo o aspecto del predicado impide asignar relevancia respecto del momento de enunciación $y$, finalmente, cuando su posición sintáctica sugiere un alcance 
que excede al del predicado y le permite funcionar como marcador extraproposicional.

En "Consideración del uso de la cláusula ditransitiva en el discurso", Claudia Borzi parte de una revisión crítica de los distintos tratamientos que ha recibido la transitividad (y la ditransitividad) en la literatura hispánica y tipológica, para enfocarse en el funcionamiento discursivo de las cláusulas ditransitivas con el verbo dar. A partir de un corpus de relatos literarios, la autora analiza el estatus de los tres participantes argumentales de estas cláusulas. Más allá de comprobar la mayor continuidad y persistencia (Givón 1983) del agente y el dativo frente al paciente, se argumenta que, en la cláusula con dar, el paciente funciona como un lugar de contacto a partir del cual agente y paciente se reconceptualizan. En este sentido, la cláusula ditransitiva se opone a la transitiva, en las que el paciente funciona como un "punto de llegada". Es decir, en contraste con el modelo cognitivista que ve la cláusula ditransitiva como cadena de acción lineal y sucesiva (Langacker 1991), en esta propuesta la cláusula ditransitiva se concibe como un triángulo, y el paciente de la cláusula con dar como el vértice en el que los otros dos argumentos entran en contacto para continuar su trayectoria discursiva resignificados.

En "Las construcciones escindidas como recurso pragmático para la expresión y configuración de la polémica en dos discursos sobre la izquierda", Nélida Murguía aborda la función argumentativa de las construcciones escindidas. Específicamente, la autora revela de qué manera el valor general de focalización contrastiva asociado con distinto tipo de construcciones escindidas es puesto al servicio de la organización polémica del discurso en contextos en los que el enunciador busca orientar la confrontación de puntos de vista. El análisis está basado en dos entrevistas a representantes de la izquierda mexicana: uno de postura anarquista y otro con orientación social-demócrata. Ambos entrevistados no solamente recurren al uso de estructuras escindidas con notable frecuencia sino que, muy significativamente, lo hacen en contextos en los que buscan articular diversos antagonismos: entre izquierda vs. no izquierda, revolución vs. reforma y anticapitalismo vs. antineoliberalismo. El capítulo resalta el potencial que ofrecen las estructuras escindidas para el análisis del discurso político, en la medida en que permiten observar la puesta en escena de voces que se confrontan, actualizando una dimensión polémica, a partir de la cual se construye la posición del enunciador.

En "La marcación de foco en el habla dirigida a niños: marcos léxicos y estrategias prosódicas”, Laura Cristina Villalobos Pedroza estudia las pistas léxicas y prosódicas que utilizan los adultos que interactúan con niños, y de qué manera estas pistas participan en el establecimiento de la atención conjunta y la construcción de la base común (common ground). A partir la base de datos ETAL 
(Rojas-Nieto 2007), consistente en interacciones espontáneas en el entorno cotidiano de los niños, el estudio selecciona 118 emisiones de seis adultos en su interacción con tres niñas de entre 23 y 29 meses. La autora estudia aquellas emisiones en que los adultos codifican un elemento focal, y analiza no solamente las características prosódicas que se asocian con este elemento (acentos tonales, tonos de juntura, excursión tonal y alineamiendo del pico tonal), sino el contexto construccional en el que aparece. En efecto, antes de codificar el elemento en foco, los adultos a menudo despliegan distintas estrategias léxicas para garantizar la atención conjunta (vocativos, actos directivos con verbos de percepción), así como para anclar la emisión al contexto físico compartido (expresiones deícticas). Entre otros resultados, el estudio muestra que, en ausencia de atractores de atención y marcadores de anclaje deíctico, el foco recibe un despliegue prosódico especial; es decir, los adultos parecen dedicar un mayor esfuerzo articulatorio a los focos que no aparecen señalados por medios léxicos, lo que sugiere que la marcación del foco no es categórica, sino que se construye en línea y a través de una variedad de recursos.

Finalmente, en "El rol de la jerarquía remática en la comprensión de oraciones con distinta configuración sintáctico-semántica”, Carolina A. Gattei, Luis A. París y Diego Shalom utilizan una tarea de lectura de textos mediante la técnica de seguimiento de movimiento oculares (eye tracking) para investigar el efecto que tiene sobre el procesamiento de oraciones la interacción entre distintas jerarquías que afectan el orden de constituyentes en español: la sintáctica, que predice el orden sujeto-objeto; la semántica, que predice el orden experimentante-estímulo; y la pragmática, que predice el orden dado-nuevo. A partir de la medición de tiempos de lectura total, tiempos de respuesta y porcentaje de aciertos en preguntas relacionadas con la interpretación del evento descrito en las oraciones usadas como estímulo, los autores muestran una influencia de la jerarquía pragmática en el costo cognitivo relacionado con la lectura de los textos y en las estrategias utilizadas por los lectores para realizar la tarea de comprensión de manera exitosa. En particular, los resultados de la tarea experimental muestran que los participantes requirieron significativamente más tiempo para leer los textos cuando la oración blanco violaba la jerarquía pragmática, independientemente de si esta oración respetaba o no el orden de constituyentes canónico.

\section{Agradecimientos}

Queremos agradecer especialmente a los colegas que aceptaron servir como dictaminadores de las distintas contribuciones, y con enorme compromiso y generosidad nos ayudaron a mejorar nuestro trabajo. Nuestro gran reconocimiento va 
para Martín Califa, Belén Carpio, Rosa María Ciscomani, Paola Cúneo, Juliana De la Mora, Josefina García Fajardo, Lilián Guerrero, Sara Gómez Seibane, Manuel Leonetti, Brook Lillehaugen, Ricardo Maldonado, Rosa Montes, Teresa Oteíza, María Piñango, Asela Reig, y un par de colegas más que prefirieron permanecer anónimos. En relación con el proceso de publicación, queremos expresar nuestro aprecio por el gran profesionalismo del equipo de De Gruyter, y en especial a Christine Lembrecht por todo el seguimiento del proyecto y a Gabrielle Cornefert por su dedicación en la corrección editorial.

Este volumen se enmarca en un proyecto de investigación sobre Interfaz Sintaxis-Pragmática a cargo de la editora y financiado por el Consejo Nacional de Ciencia y Tecnología de México (CONACYT Ciencia Básica 0256785). La asistencia a distintos foros y reuniones de trabajo donde tuvimos oportunidad de compartir ideas y estrechar lazos de colaboración con varios de los autores de los capítulos que aquí se presentan, también ha sido posible por el generoso apoyo de la Facultad de Lenguas y Letras de la Universidad Autónoma de Querétaro. Para ambas instituciones, nuestro agradecimiento.

\section{Referencias}

Casielles-Suárez, Eugenia. 2004. The Syntax-Information Structure Interface: Evidence from Spanish and English. New York: Routledge.

Chafe, Wallace (ed.). 1980. The Pear Stories: Cognitive, Cultural, and Linguistic Aspects of Narrative Production. Norwood: Ablex.

Cresti, Emanuela y Massimo Moneglia (eds). 2005. C-ORAL-ROM. Integrated Reference Corpora for Spoken Romance Languages. Amsterdam: John Benjamins.

Dik, Simon. 1989. The Theory of Functional Grammar. Part I. Dordrecht: Foris.

Dik, Simon. 1997. The Theory of Functional Grammar. Part II. Dordrecht: Foris.

Dik, Simon, Maria Hoffman, Jan de Jong, Sie Ing Djang, Harry Stroomer y Lourens de Vries. 1981. On the typology of focus phenomena. En Teun Hoekstra (ed.). Perspectives on Functional Grammar, 41-74. Dordrecht: Foris.

Erteschik-Shir, Nomi. 1997. The Dynamics of Focus Structure. Cambridge: Cambridge University Press.

Givón, Talmy. 1983. Topic continuity in discourse. A quantitative cross-language study. Amsterdam: John Benjamins.

Karttunen, Lauri. 1976. Discourse referents. En James D. McCawley (ed.). Syntax and Semantics, vol. 7. 363-386, New York: Academic Press.

Lakoff, George. 1987. Women, fire and dangerous things: What categories reveal about the mind. Chicago: University of Chicago Press.

Lambrecht, Knud. 1994. Information Structure and Sentence Form: Topic, Focus, and the Mental Representations of Discourse Referents. Cambridge: Cambridge University Press. 
Langacker, Ronald. 1991. Foundations of cognitive grammar. Descriptive application. Stanford: Stanford University Press.

Leonetti, Manuel. 2011. Indefinidos, nombres escuetos y clíticos en las dislocaciones en español. Cuadernos de la ALFAL 3. 100-123.

Matić, Dejan. 2015. Information Structure in Linguistics. En James Wright (ed.). International Encyclopedia of the Social \& Behavioral Sciences, 2nd edition, volume 12. 95-99. Oxford: Elsevier.

Matić, Dejan y Daniel Wedgwood. 2013. The meanings of focus: The significance of an interpretation-based category in cross-linguistic analysis. Journal of Linguistics 49(1). $127-163$.

Olarrea, Antxon. 2012. Word Order and Information Structure. En José Ignacio Hualde, Antxon Olarrea y Erin 0'Rourke (eds.). The Handbook of Spanish Linguistics, 603-628. Oxford: Blackwell.

Ozerov, Pavel. 2015. Information structure without topic and focus: Differential object marking in Burmese. Studies in Language 39(2). 386-423.

Reinhart, Tanya. 1981. Pragmatics and Linguistics: An Analysis of Sentence Topics, Philosophica 27(1). 53-94.

Rojas-Nieto, Cecilia. 2007. La base de datos ETAL (Etapas tempranas en la adquisición del lenguaje). En Alejandra Vigueras (ed.). Jornadas filológicas 2005: Memoria, 575-599. México: UNAM-IIFL.

Sasse, Hans-Jürgen. 1995. Theticity and VS order: a case study. Sprachtypologie und Universalienforschung (STUF) 48. 3-31.

Skopetas, Stavros, Ines Fiedler, Sam Hellmuth, Anne Schwarz, Ruben Stoel, Gisbert Fanselow, Caroline Féry y Manfred Krifka. 2006. Questionnaire on Information Structure (QUIS). Reference Manual. Potsdam: Universität Potsdam.

Vallduví, Enric. 1992. The informational component. New York: Garland.

van Valin, Robert. 1999. A Typology of the Interaction of Focus Structure and Syntax. En Ekatarina Raxilina and Jakov Testelec (eds.). Typology and the Theory of Language: From Description to Explanation. Moscow: Languages of Russian Culture.

van Dijk, Teun. 1981. Sentence topic and discourse topic. En Teun van Dijk (ed.), Studies in the pragmatics of discourse, 177-193. Paris: Mouton.

von Heusinger, Klaus. 1999. Intonation and Information Structure. Habilitationsschrift. University of Konstanz.

Ward, Gregory y Betty Birner. 2004. Information Structure and Non-Canonical Syntax. En Laurence Horn y Gregory Ward (eds.). The Handbook of Pragmatics, 153-174. Oxford: Blackwell. 